\title{
OPTIMAL TEMPERATURE PATHS \\ FOR THERMORHEOLOGICALLY SIMPLE VISCOELASTIC MATERIALS WITH CONSTANT POISSON'S RATIO ARE CANONICAL*
}

\author{
BY \\ MORTON E. GURTIN (Carnegie-Mellon University) \\ AND \\ LEA F. MURPHY (Oregon State University)
}

\begin{abstract}
In this note we discuss the thermal stress problem for a thermorheologicallysimple linearly-viscoelastic body, subjected to a spatially-uniform temperature field and homogeneous boundary conditions, assuming that Poisson's ratio is constant and inertia negligible. In particular, we consider the following optimization problem: of all temperature paths $\theta(t), 0 \leqslant t \leqslant t_{f}$, which belong to a given function class, is there one which renders a given stress measure a minimum at time $t_{f}$. We show that a resulting optimal path $\theta(t)$ (if it exists) is canonical: $\theta(t)$ is independent of the shape of the body and of the particular homogeneous boundary conditions.
\end{abstract}

The viscoelastic problem. We consider a thermorheologically-simple viscoelastic material subject to a spatially-uniform temperature field. We assume that Poisson's ratio $\nu$ is constant. The stress $\mathbf{S}(\mathbf{x}, t)$, strain $\mathbf{E}(\mathbf{x}, t)$, and temperature $\theta(t)$ are then related through the constitutive equations ${ }^{2}$

$$
\begin{aligned}
\mathbf{S}(\mathbf{x}, t) & =\int_{0}^{t} G(\xi(t)-\xi(s)) \dot{\mathbf{Q}}(\mathbf{x}, s) d s, \\
\mathbf{Q} & =\mathbf{E}+\frac{1}{1-2 \nu}(\operatorname{tr} \mathbf{E}) \mathbf{I}-\frac{(1+\nu) \alpha}{1-2 \nu} \eta \mathbf{I},
\end{aligned}
$$

with $G(t)$ the scalar relaxation function, $\alpha$ the coefficient of termal expansion,

\footnotetext{
* Received January 18, 1983. This study was supported by the Army Research Office and the National Science Foundation.

'Actually, $\theta$ is the temperature increment relative to a fixed reference temperature.

${ }^{2}$ Cf., e.g., Muki and Sternberg [1].

${ }^{3}$ We use the following notation (cf., e.g., Gurtin [2]): upper-case boldface letters are second-order tensors; $\mathbf{A}^{T}$ and tr $\mathbf{A}$ are the transpose and trace of $\mathbf{A} ;|\mathbf{A}|=\left(A_{i j} A_{i j}\right)^{1 / 2}$ (summation over repeated subscripts implied); $\mathbf{I}$ is the identity; $\operatorname{div} \mathbf{S}$ is the vector field with components $\partial S_{i j} / \partial x_{j} ; \nabla \mathbf{u}$ is the tensor field with components $\partial u_{i} / \partial x_{j}$.
} 


$$
\xi(t)=\int_{0}^{t} \varphi(\theta(\beta)) d \beta
$$

the reduced time, and $\varphi(\theta)$ the shift factor.

As a customary, we assume quasi-static conditions; the remaining field equations then take the form

$$
\operatorname{div} \mathbf{S}=\mathbf{0}, \quad \mathbf{E}=\frac{1}{2}\left(\nabla \mathbf{u}+\nabla \mathbf{u}^{T}\right)
$$

with $\mathbf{u}(\mathbf{x}, t)$ the displacement. Here and in what follows the time is confined to the interval $0 \leqslant t<\infty$, it being tacit that $\mathbf{u}, \mathbf{E}, \mathbf{S}$, and $\theta$ all vanish for $t<0$.

We limit our attention to the homogeneous boundary conditions

$$
\mathbf{u}=\mathbf{0} \quad \text { on } \mathcal{S}_{1}, \quad \mathbf{S n}=\mathbf{0} \text { on } \mathcal{S}_{2},
$$

where $\delta_{1}$ and $\delta_{2}$ are complementary subsets of - and $\mathbf{n}$ the outward unit normal to - the boundary of the body $\Re$.

The boundary-value problem (1)-(4) can be solved, at least formally, as follows. Write (1), in the form

$$
\mathbf{S}=G \# \mathbf{Q} \text {. }
$$

Since $\theta$ is independent of position, the operation \# commutes with spatial differentiation. Hence (3) ${ }_{2}$ becomes

$$
G \#(\operatorname{div} \mathbf{Q})=\mathbf{0},
$$

which is satisfied by taking

$$
\operatorname{div} \mathbf{Q}=\mathbf{0} .
$$

Similarly, the boundary condition $(4)_{2}$ is satisfied provided

$$
\text { Qn }=\mathbf{0} \text { on } \delta_{2} \text {. }
$$

Our problem thus reduces to solving the linear thermoelastic problem defined by the field equations (1) $)_{2},(3)_{2}$, and (6) and the boundary conditions (4) $)_{1}$ and (7). Let $\mathbf{Q}=\mathbf{P}(\mathbf{x})$ denote the solution to the boundary-value problem corresponding to $\theta(t)=1$, for all $t$. Since the boundary data are null and the field equations linear, the solution of the thermoelastic problem is ${ }^{4}$

$$
\mathbf{Q}(\mathbf{x}, t)=\theta(t) \mathbf{P}(\mathbf{x})
$$

hence (5) yields

$$
\begin{aligned}
\mathbf{S}(\mathbf{x}, t) & =\tau(t) \mathbf{P}(\mathbf{x}), \\
\tau & =G \# \theta .
\end{aligned}
$$

Optimal temperature paths. Assume we are given a (spatial) stress measure $\Sigma(\mathbf{A})$ defined for all sufficiently nice (symmetric, second-order) tensor functions $\mathbf{A}(\mathbf{x})$ on $\mathscr{B}$. Regarding the properties of $\Sigma$, we need only suppose that $\Sigma \geqslant 0$ and that

$$
\Sigma(\beta \mathbf{A})=|\beta| \Sigma(\mathbf{A})
$$

\footnotetext{
${ }^{4}$ We assume the data such that both elastic and viscoelastic problems are well posed (in the sense of existence and uniqueness).
} 
for any scalar constant $\beta$. Examples of stress measures are the $L^{2}$-norm

$$
\Sigma(\mathbf{A})=\left[\int_{\mathscr{B}}|\mathbf{A}|^{2} d V\right]^{1 / 2}
$$

the sup-norm

$$
\Sigma(\mathbf{A})=\sup _{x \in \mathscr{B}}|\mathbf{A}(\mathbf{x})|
$$

the $\mathbf{x}_{0}$-evaluation of $|\mathbf{A}|\left(\mathbf{x}_{0}\right.$ a particular point of $\left.\mathscr{B}\right)$

$$
\Sigma(\mathbf{A})=\left|\mathbf{A}\left(\mathbf{x}_{0}\right)\right| ;
$$

the $L^{2}$-norm, sup-norm, or $\mathbf{x}_{0}$-evaluation of either the octahedral shear stress or the principal-stress magnitude corresponding to $\mathbf{A}$.

The optimization problem under consideration consists in finding temperature paths that minimize the associated stress measure. ${ }^{5}$ To state this problem succinctly, let us agree to use the term temperature path for a function $\theta(t), 0 \leqslant t \leqslant t_{f}$, which belongs to some prescribed function class $\mathscr{F}$. For example, $\mathscr{F}$ may be the set of all sufficiently regular functions that have $\theta(0)$ and $\theta\left(t_{f}\right)$ equal to given initial and final temperatures. Given a temperature path, we write

$$
\mathbf{S}_{f}(\mathbf{x})=\mathbf{S}\left(\mathbf{x}, t_{f}\right), \tau_{f}=\tau\left(t_{f}\right)
$$

for the corresponding functions computed using (8). (Here and in what follows $t_{f}>0$ and $\mathscr{F}$ are fixed.)

OPTIMIZATION PROBLEM. Find a temperature path $\theta(t)$ that minimizes the stress measure $\Sigma\left(\mathbf{S}_{f}\right)$.

By $(8)$, and (9),

$$
\Sigma\left(\mathbf{S}_{f}\right)=\left|\tau_{f}\right| \Sigma(\mathbf{P})
$$

If

$$
\Sigma(\mathbf{P})=0,
$$

then all temperature paths are optimal; we henceforth exclude from our discussion situations in which (11) is satisfied. We then conclude from (10) that the optimization problem is equivalent to the

REDUCED OPTIMIZATION PROBLEM. Find a temperature path $\theta(t)$ that minimizes $\left|\tau_{f}\right|$; i.e., that minimizes the functional

$$
\left|\int_{0}^{t_{f}} G\left(\int_{s}^{t_{f}} \varphi(\theta(\beta)) d \beta\right) \dot{\theta}(s) d s\right| .
$$

\footnotetext{
${ }^{5}$ This problem - within an essentially one-dimensional framework - was studied by Weitsman and Ford [3]. Weitsman [4], and Gurtin and Murphy [5]. Problems of this type arise, for example, in the curing of polymeric materials (cf. [3-5]).

${ }^{6}$ Generally, solutions of this problem will not be everywhere differentiable [3-5], so that (12) must be interpreted in some generalized sense (cf. [5]).
} 
A direct consequence of this equivalence is the following interesting result: the optimal path, if it exists, is independent of the shape of the body, of the particular homogeneous boundary conditions, and of the particular stress measure.

The foregoing equivalence is important as it allows one to determine the optimal temperature path without solving the underlying boundary-value problem.

Actually, a slightly stronger result is possible. Let us agree to call a temperature path $\hat{\theta}$ better than a temperature path $\breve{\theta}$ provided

$$
\Sigma\left(\hat{\mathbf{S}}_{f}\right) \leqslant \Sigma\left(\tilde{\mathbf{S}}_{f}\right)
$$

(using obvious notation). It then follows from (10) that: if $\hat{\theta}$ is better than $\tilde{\theta}$ for a given body under given homogeneous boundary conditions, $\hat{\theta}$ is better than $\tilde{\theta}$ for any body under all such boundary conditions, and for any choice of stress measure.

This result is important experimentally as it shows that a "good" temperature path determined experimentally using a configuration amenable to testing is "good" for bodies of all shapes.

\section{REFERENCES}

[1] R. Muki and E. Sternberg, On transient thermal stresses in viscoelastic materials with temperature-dependent properties, J. Appl. Mech. 83, 193-207 (1961)

[2] M. E. Gurtin, An introduction to continuum mechanics, Academic Press, New York, 1981

[3] Y. Weitsman and D. Ford, On the optimization of cool-down temperatures in viscoelastic resins, Proc. 14th Mtg. Soc. Engin. Sci., 323-339 (1977)

[4] Y. Weitsman, Optimal cool-down in linear viscoelasticity, J. Appl. Mech. 47, 35-39 (1980)

[5] M. E. Gurtin and L. F. Murphy, On optimal temperature paths for thermorheologically simple viscoelastic materials Quart. Appl. Math. 38, 179-189 (1980) 